\title{
Low-level percepts predict emotion concepts across modalities and cultures
}

\author{
Beau Sievers ${ }^{1 \oplus}$, Carolyn M. Parkinson ${ }^{2 \odot}$, Trent Walker ${ }^{3}$, William Haslett ${ }^{4}$, \& Thalia Wheatley ${ }^{1}$ \\ ${ }^{1}$ Department of Psychological and Brain Sciences, Dartmouth College, Hanover, NH 03755 \\ ${ }^{2}$ Department of Psychology, University of California, Los Angeles, CA 90095 \\ ${ }^{3}$ Group in Buddhist Studies, University of California, Berkeley, CA 94720 \\ ${ }^{4}$ Department of Biomedical Data Science, Geisel School of Medicine at Dartmouth, Hanover, NH \\ 03755 \\ ๑Authors contributed equally.
}

\begin{abstract}
Köhler (1929) first demonstrated that "hard" sounds (e.g., takete) are intuitively associated with spiky shapes, while "soft" sounds (e.g., maluma) are associated with rounded shapes. Here we replicate Köhler's original shape-sound correspondence study using non-phonetic sounds in a population with little exposure to globalized culture. Further, we show that these low-level crossmodal correspondences predict judgments of emotional arousal. Together, these findings suggest that cross-cultural, high-level concepts may piggyback on lowlevel perceptual correspondences.
\end{abstract}

\section{Introduction}

In a famous demonstration of correspondences between sound and vision, Köhler (1929) asked participants to match the nonsense words takete and maluma to two figures: one round and the other spiky. He reported that answers occurred "without hesitation," but did not report the results. In context, it is clear he believed the outcome was so obvious it need not be stated explicitly: takete matched the spiky shape and maluma the rounded. Köhler's finding is robust. It has been replicated directly as the Bouba-Kiki effect (Ramachandran \& Hubbard, 2001), cross-culturally (Bremner et al., 2013; Davis, 1961; cf. Rogers \& Ross, 1975), in toddlers (Maurer, Pathman, \& Mondloch, 2006) and in infants (Ozturk, Krehm, \& Vouloumanos, 2013).

We tested whether Köhler's effect extends to concepts of emotional arousal. Our studies were conducted in L'ak, a remote Kreung village located in northeastern Cambodia, then replicated at Dartmouth College in the United States. We chose L'ak because of its high degree of geographic and cultural isolation. If crossmodal correspondence effects occur in a remote population, then they are not limited to or caused by the norms and customs of globalized culture. Further description of L'ak is included in the Supplementary Material, as well as Sievers et al. (2013) and Parkinson et al. (2012). Taken together, the studies presented here provide insight into how high-level concepts like 
emotional arousal can arise from unimodal low-level building blocks extracted from auditory and visual inputs.

\section{Study 1: Sound-shape correspondence}

\section{Methods}

Participants in all studies were native Kreung speakers who lived in L'ak or members of the Dartmouth College community in the US. Studies conducted in L'ak were approved by the Ratanakiri Ministry of Culture and the L'ak village head. Ethical approval for all US studies was obtained from the Dartmouth College institutional review board. See Supplementary Material for a complete description of recruiting practices. Sample sizes in L'ak were limited by the availability of participants. Sample sizes in the US were set using a power analysis (see Supplementary Material).

Shapes. Previous studies of sound-shape correspondence have used spiky and rounded shapes that differed on one or more parameters not relevant to the experimental manipulation. To test for the effect of spikiness alone, we created shape pairs based on Köhler's (1929) original shapes, but with the general shape, relative positions of line crossings, and size matched for each pair (Figure 1).

Sounds. We used two non-phonetic sounds with different acoustic characteristics. ${ }^{1}$ One sound, designed to match takete or the spiky shape, consisted of four bursts of white noise in an asymmetrical rhythm. The second sound, designed to match maluma or the rounded shape, consisted of a sine wave at $250 \mathrm{~Hz}$ with amplitude modulation in a rhythm similar to the noise burst pattern (Figure 1). Both sounds were 1.5s. Because the perceived volume of white noise bursts vs. sine waves does not correspond, the volume levels of the sounds were subjectively matched. ${ }^{2}$ In all studies sounds were presented using closed-back, Sony MDR-7506 headphones. Volume levels were calibrated so only participants (and not the experimenter) could hear the sounds.

\footnotetext{
${ }^{1}$ We attempted to directly replicate Köhler (1929), but found that the nonsense words takete and maluma were heard as groups of meaningful monosyllabic words in Kreung (a West Bahnaric language with phonology unlike English; Keller, 2001). This rendered the task absurd: pilot participants laughed and asked why we thought these squiggly lines had anything to do with the words we were speaking. Possibly explaining this null result, Westbury (2005) has shown that crossmodal correspondences are disrupted for meaningful (versus nonsense) words, and Styles \& Gawne (2017) have shown similar disruptions when the nonsense words used do not fit the sound structure of the target language.

${ }^{2}$ Volume levels were matched by author BS who has training and professional experience as an audio mixing and mastering engineer.
} 
Participants heard one sound in headphones and were shown one shape pair. They were asked to point to (in L'ak) or click on (in the US) the shape that best matched the sound. Shapes and sounds were counterbalanced across participants.
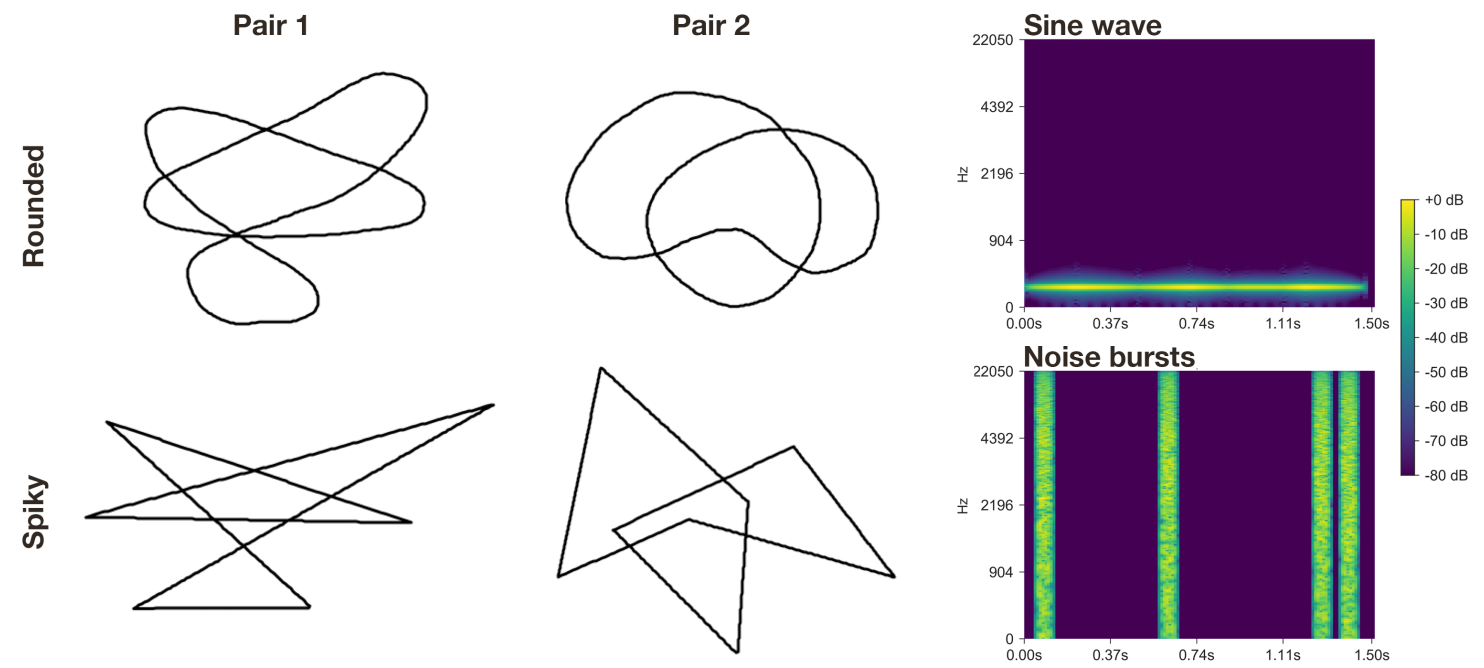

Figure 1: Left and center: Matched shape pairs. The spiky shape in Pair 1 and the rounded shape in Pair 2 were used in Köhler (1929). Right: Spectrograms of sound stimuli.

\section{Results}

All results are summarized in Figure 2. Exact binomial tests showed that participants in L'ak chose the congruent shape $83 \%$ of the time $(95 \% \mathrm{Cl}$ : .64$.94, \mathrm{p}<.001, \mathrm{~N}=29$ ), while participants in the US chose the congruent shape $97 \%$ of the time (95\% Cl: .89-1.0, $\mathrm{p}<.001, \mathrm{~N}=61)$. This cross-culturally replicated Köhler's (1929) finding using non-phonetic sounds, providing evidence that crossmodal correspondences depend on features that are common across shapes, phonetic, and non-phonetic sounds.

\section{Study 2: Shape-emotion and sound-emotion correspondence}

\section{Methods}

Emotional states are often described using language that equally fits shapes: an irritable person is "prickly," an empathetic person is "soft," and so on. Likewise, shapes are used to communicate emotion: death metal bands use elaborate, violently spiky logotypes, while romantic poetry is written in flowing, flowery curlicues. Previous research conducted in the United States has shown that minimal, abstract line drawings, similar to those used by Köhler (1929), reliably express emotional states (Lundholm, 1921; Poffenberger \& Barrows, 1924). 
Study 2 tests whether the link between sounds and shapes shown in Study 1 extends to high-level concepts of emotional arousal in a remote population.

In the sound-emotion task participants heard both sounds in headphones and were asked which sound best corresponded with Anger and which with Sadness. In the shape-emotion task, participants were shown a shape pair and asked the same question. In L'ak, all participants $(N=37)$ completed both tasks, with task and stimulus order counterbalanced. In the US, each task was completed by a different group of participants (shape-emotion $N=60$; soundemotion $N=63$ ). In all tasks in both locations experimenters were blind to the presentation order of the stimuli and which shape or sound the participant selected.

\section{Results}

All results are summarized in Figure 2. All reported statistics are exact binomial tests. The sound-emotion congruence rate was $76 \%$ in L'ak (95\% Cl: .61-.88, $N=37)$, and $82 \%$ in the US (95\% Cl: .71-.91, $\mathrm{p}<.001, N=63)$. The shape-emotion congruence rate was $68 \%$ in L'ak $(95 \% \mathrm{Cl}: .53-.82, N=37)$ and $98 \%$ in the US (95\% Cl: .91-1.0, $\mathrm{p}<.001, N=60)$. Participants associated spiky shapes and noise bursts with high arousal (Angry), and rounded shapes and the sine wave with low arousal (Sad).

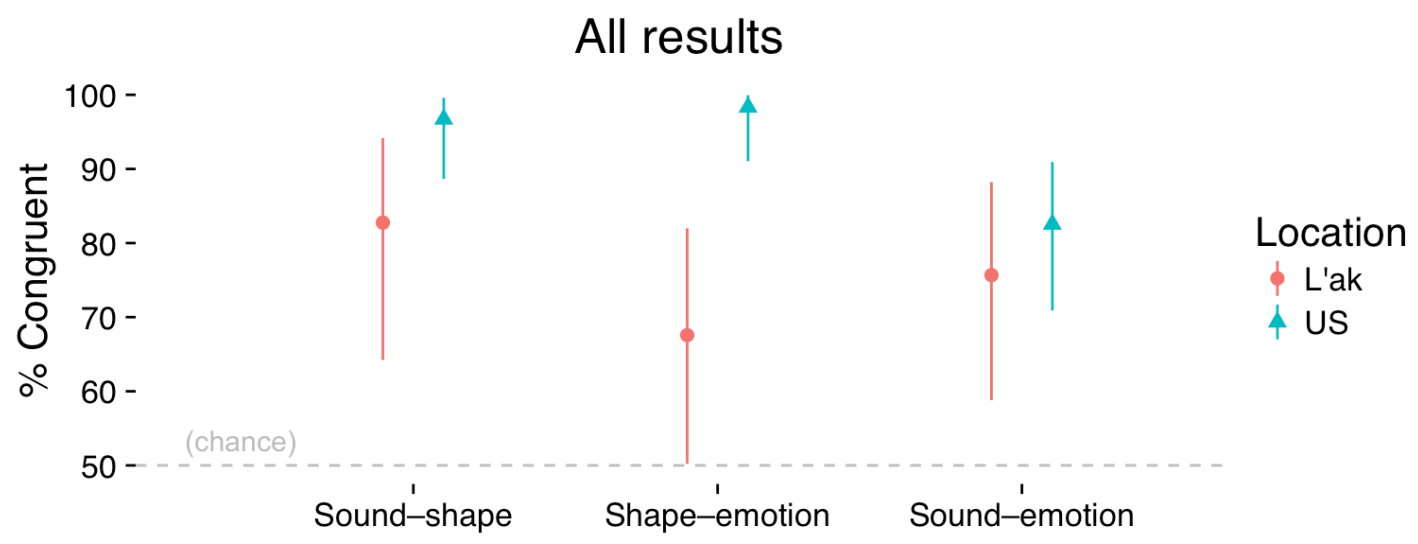

Figure 2. Estimates and 95\% confidence intervals for all results. The spiky shape was associated with noise bursts and the rounded shape with the sine wave. Angry was associated with noise bursts and spiky shapes while Sad was associated with the sine wave sound and rounded shapes. For all tasks, chance was $50 \%$. 


\section{Discussion}

All participants, in the US and L'ak, associated a noise burst sound with spiky shapes and a sine wave sound with rounded shapes. Further, different levels of emotional arousal were associated with shapes and sounds in the same way: Angry with spiky shapes and the noise burst sound, and Sad with rounded shapes and the sine wave sound.

Crossmodal correspondences have been documented for almost a century, yet why they occur is poorly understood. One possibility is that features common to sounds and shapes are represented the same way in the brain. Such a shared representation could facilitate predictive coding and statistical learning processes (Clark, 2013; Spence \& Deroy, 2012, 2013), efficiently solving the problem of rapidly identifying crossmodal similarities. Sievers et al. (2017) show that the spectral centroid (the "center of gravity" of a frequency spectrum) is a promising candidate feature that could be readily represented across modalities, supporting crossmodal correspondences.

It is well-established that physical arousal constrains human behavioral patterns: people in a high arousal state produce spikier speech and movement (Banse \& Scherer, 1996; Pollick, Paterson, Bruderlin, \& Sanford, 2001; Tooby \& Cosmides, 1990). Sensitivity to these features and similar crossmodally redundant signals should improve signal detection and thereby reproductive fitness. Although the present study is limited to human participants, we expect these principles to operate similarly in other species. Supporting this conjecture, Filippi et al. (2017) recently demonstrated that low-level acoustic properties of animal calls communicate arousal across species. Our data suggest that these patterns also manifest in the visual domain (e.g., movement) and are similarly exploited to predict the behavioral patterns of conspecifics.

The present results suggest that low-level perceptual correspondences play a role in high-level concept formation, thereby shedding light on how humans communicate despite linguistic and cultural barriers. Developing a deeper understanding of crossmodal correspondences will further reveal how these communicative abilities may have arisen from our evolutionary history.

\section{Acknowledgements}

We thank the Ratanakiri Ministry of Culture, Ockenden Cambodia, and Cambodian Living Arts for assistance in Cambodia. This research was supported in part by a McNulty Grant from the Nelson A. Rockefeller Center (TW), a Foreign Travel Award from the John Sloan Dickey Center for International Understanding (TW), a Natural Sciences and Engineering Research Council of Canada Postgraduate Scholarship (CP), and Neukom Institute for Computational Science Graduate Fellowships (BS and CP). 


\section{Author Contributions}

C. Parkinson, B. Sievers, and T. Wheatley contributed equally to study design. W. Haslett created the matched shape pair stimuli. B. Sievers performed the data analyses in consultation with C. Parkinson. B. Sievers, T. Wheatley, and C. Parkinson wrote the paper. T. Walker provided vital assistance adapting experimental designs for use with translators and helped collect data.

\section{References}

Banse, R., \& Scherer, K. R. (1996). Acoustic profiles in vocal emotion expression. Journal of Personality and Social Psychology, 70(3), 614-36. Retrieved from http://www.ncbi.nlm.nih.gov/pubmed/8851745

Bremner, A. J., Caparos, S., Davidoff, J., de Fockert, J., Linnell, K. J., \& Spence, C. (2013). "Bouba" and "Kiki" in Namibia? A remote culture make similar shape-sound matches, but different shape-taste matches to Westerners. Cognition, 126(2), 165-72. http://doi.org/10.1016/j.cognition.2012.09.007

Clark, A. (2013). Whatever next? Predictive brains, situated agents, and the future of cognitive science. The Behavioral and Brain Sciences, 36(3), 181204. http://doi.org/10.1017/S0140525X12000477

Davis, R. (1961). The fitness of names to drawings. A cross-cultural study in Tanganyika. British Journal of Psychology, 52(3), 259-268. Retrieved from http://onlinelibrary.wiley.com/doi/10.1111/j.20448295.1961.tb00788.x/abstract

Filippi, P., Congdon, J. V., Hoang, J., Bowling, D. L., Reber, S. A., Pašukonis, A., ... Güntürkün, O. (2017). Humans recognize emotional arousal in vocalizations across all classes of terrestrial vertebrates: evidence for acoustic universals. Proceedings of the Royal Society of London B: Biological Sciences, 284(1859), 1-9. Retrieved from http://rspb.royalsocietypublishing.org/content/284/1859/20170990?etoc

Keller, C. E. (2001). Brao-Krung phonology. Mon-Khmer Studies, 31, 1-13. Retrieved from http://sealang.net/sala/archives/pdf8/keller2001brao.pdf

Köhler, W. (1929). Gestalt Psychology. Oxford, England: Liveright.

Lundholm, H. (1921). The affective tone of lines: experimental researches. Psychological Review, 28(1), 43-60.

Maurer, D., Pathman, T., \& Mondloch, C. J. (2006). The shape of boubas: soundshape correspondences in toddlers and adults. Developmental Science, 9(3), 316-22. http://doi.org/10.1111/j.1467-7687.2006.00495.x

Ozturk, O., Krehm, M., \& Vouloumanos, A. (2013). Sound symbolism in infancy: 
evidence for sound-shape cross-modal correspondences in 4-month-olds. Journal of Experimental Child Psychology, 114(2), 173-86. http://doi.org/10.1016/j.jecp.2012.05.004

Parkinson, C., Kohler, P. J., Sievers, B., \& Wheatley, T. (2012). Associations between auditory pitch and visual elevation do not depend on language: Evidence from a remote population. Perception, 41(7), 854-861. http://doi.org/10.1068/p7225

Poffenberger, A., \& Barrows, B. (1924). The feeling value of lines. Journal of Applied Psychology, 8(2), 187-205.

Pollick, F. E., Paterson, H. M., Bruderlin, A., \& Sanford, A. J. (2001). Perceiving affect from arm movement. Cognition, 82, 51-61. http://doi.org/10.1016/S0010-0277(01)00147-0

Ramachandran, S., \& Hubbard, E. M. (2001). Synaesthesia-a window into perception, thought and language, (12), 3-34.

Sievers, B., Lee, C., Haslett, W., \& Wheatley, T. (2017). A supramodal code for emotional arousal. Retrieved from https://psyarxiv.com/wucs4/

Sievers, B., Polansky, L., Casey, M., \& Wheatley, T. (2013). Music and movement share a dynamic structure that supports universal expressions of emotion. Proceedings of the National Academy of Sciences of the United States of America, 110(1), 70-5. http://doi.org/10.1073/pnas.1209023110

Spence, C., \& Deroy, O. (2012). Crossmodal correspondences: Innate or learned? I-Perception, 3(5), 316-318. http://doi.org/10.1068/i0526ic

Spence, C., \& Deroy, O. (2013). How automatic are crossmodal correspondences? Consciousness and Cognition, 22(1), 245-260. http://doi.org/10.1016/j.concog.2012.12.006

Styles, S. J., \& Gawne, L. (2017). When Does Maluma/Takete Fail? Two Key Failures and a Meta-Analysis Suggest That Phonology and Phonotactics Matter. I-Perception, 8(4), 204166951772480. http://doi.org/10.1177/2041669517724807

Tooby, J., \& Cosmides, L. (1990). The past explains the present: Emotional adaptations and the structure of ancestral environments. Ethology and Sociobiology, 11(4), 375-424. http://doi.org/10.1016/0162-3095(90)90017-Z 